\title{
MUCOCUTANEOUS ADVERSE EFFECTS IN PATIENTS UNDERGOING CANCER CHEMOTHERAPY
}

\author{
Garima Sharma1 ${ }^{1}$ P. K. Nigam²
}

${ }_{1}^{1}$ Assistant Professor, Department of Skin, Pt. JNM Medical College, Raipur, Chhattisgarh, India.

2Director, Professor and HOD, Department of Skin, Pt. JNM Medical College, Raipur, Chhattisgarh, India.

\section{BACKGROUND}

ABSTRACT

New chemotherapeutic agents and new protocols in oncology have led to an increasing survival rate in patients affected by tumours. Traditional chemotherapy drugs as well as the newer targeted agents are associated with a wide array of cutaneous toxicities.[1] Toxic effects on skin, hair and nails can negatively affect the quality of life and also lead to interruption or discontinuation of these drugs.[2]

Aim- To study the various mucocutaneous adverse effects associated with cancer chemotherapy.

\section{MATERIALS AND METHODS}

A total of 200 patients with malignancies undergoing chemotherapy in the oncology ward and outpatient department were screened in this observational study. We have clinically examined 200 patients affected by cancer determining type, treatment and evolution of cutaneous side effects related to chemotherapy. It is a hospital-based observational study. All patients were counselled about the study and informed written consent was obtained. Patients were examined before start of chemotherapy treatment and after every cycle of chemotherapy. Data collected was analysed using SPSS version 16.0.

\section{RESULTS}

In this study, 200 patients including 118 females and 82 males were studied. Majority (56\%) of the patients belonged to the age group of 41 - 60 years. The common indications for chemotherapy were carcinoma breast (29\%), tongue (11\%), buccal mucosa $(10 \%)$, ovary $(8 \%)$ and stomach $(6 \%)$. Among the cutaneous adverse events noted, hair changes were the most common presentation and were reported in $156(78 \%)$ patients. Skin changes were seen in $130(65 \%)$ cases, nail changes in 102 (51\%) cases and mucosal changes in 46 (23\%) patients. Cisplatin, cyclophosphamide, 5-fluorouracil, carboplatin, paclitaxel and doxorubicin were the most frequently prescribed chemotherapeutic drugs.

\section{CONCLUSION}

Oncological therapies have become more selective and have low systemic toxicity because of their high specificity, but cutane ous side effects are common and may worsen the quality of life of these patients. Our observations necessitate a joint effort between dermatology and oncology for the early recognition and adequate treatment of the cutaneous adverse effects associated with cancer chemotherapy, which may help in reducing morbidity and improving compliance.

\section{KEY WORDS}

Chemotherapy, Skin Toxicity, Adverse Reaction.

HOW TO CITE THIS ARTICLE: Sharma G, Nigam PK. Mucocutaneous adverse effects in patients undergoing cancer chemotherapy. J. Evolution Med. Dent. Sci. 2018;7(42):4535-4538, DOI: 10.14260/jemds/2018/1012

\section{BACKGROUND}

Cancer is a leading cause of mortality and morbidity in both developed and developing parts of the world with the disease burden projected to grow exponentially in the future. Anticancer drugs usually affect rapidly growing cells and hence the skin, hair follicles and nail matrix are the frequent targets of their toxicities.[3] In recent years, targeted therapy has considerably increased survival rate in patients affected by important solid tumours of kidney, lungs, colon-rectum, breast and liver. Despite the benefits of all these chemotherapeutic agents, toxic effects on the skin may eventually result in poor compliance of patients and interruptions or discontinuation of antineoplastic therapy. ${ }^{[4,5]}$

'Financial or Other Competing Interest': None.

Submission 30-08-2018, Peer Review 26-09-2018,

Acceptance 03-10-2018, Published 15-10-2018.

Corresponding Author:

Dr. Garima Sharma

Department of Skin,

Pt. JNM Medical College, Raipur-492001,

Chhattisgarh, India.

E-mail: drsharmaji007@gmail.com

DOI: $10.14260 /$ jemds $/ 2018 / 1012$

\section{MATERIALS AND METHODS}

An observational study was conducted at our centre. A total of 200 patients diagnosed with cancer attending the Departments of Oncology and Dermatology in a tertiary care hospital between January 2018 and July 2018, who underwent chemotherapy and satisfied the inclusion and exclusion criteria were included in this study.

\section{Inclusion Criteria}

1. Patients above the age of 18 years and who gave consent for the study were included in the study.

2. Patients of both sexes who suffered from mucocutaneous adverse effects, which began after initiation of the anticancer drug.

\section{Exclusion Criteria}

1. Patients on radiotherapy.

2. Patients below the age of 18 years.

3. Patients who developed dermatological manifestations as a result of internal malignancy or before starting of chemotherapy.

All patients were counselled about the study and informed written consent was obtained. All patients underwent a complete dermatological examination. 
Information including demographic data, cancer type, chemotherapeutic agents used, any significant dermatologic history and ongoing dermatologic treatment was collected using a structured proforma. Detailed descriptions of the clinical features as well as characterisation of the severity and evolution of cutaneous lesions were assessed and documented.Patients were examined before the start of chemotherapy treatment and at each clinical visit for every cycle of chemotherapy and the lesions were photographed. Data collected were analysed using SPSS version 16 (SPSS Inc. Chicago, USA).

Cutaneous lesions were diagnosed on the basis of typical clinical manifestations.

\section{RESULTS}

Out of 200 cancer patients studied, 82 (41\%) were males and 118 (59\%) were females. [Graph 1] They had 28 different types of malignancies.

Breast cancer was the most common cancer seen in 58 (29\%) patients followed by tongue cancer in 22 (11\%) patients, buccal mucosa cancer in $20(10 \%)$ patients and ovarian cancer in $16(8 \%)$ patients. The remaining types are depicted in figure below [Graph 2].

Common chemotherapeutic drugs used in this study were: cetuximab, gefitinib, imatinib, sorafenib, paclitaxel, vincristine, vinblastine, 6-mercaptopurine, 5-fluorouracil, cytarabine, capecitabine, gemcitabine, cisplatin, carboplatin, oxaliplatin, etoposide, cyclophosphamide, doxorubicin, daunorubicin, epirubicin, hydroxyurea, CHOP (cyclophosphamide, doxorubicin, vincristine, prednisolone) and ABVD (doxorubicin, bleomycin, vinblastine, dacarbazine) regimens.

Hair changes were the most common adverse effect noticed in $156(78 \%)$ patients followed by skin changes in 130 (65\%), nail changes in $102(51 \%)$ and mucosal changes in $46(23 \%)$ patients [Graph 3].

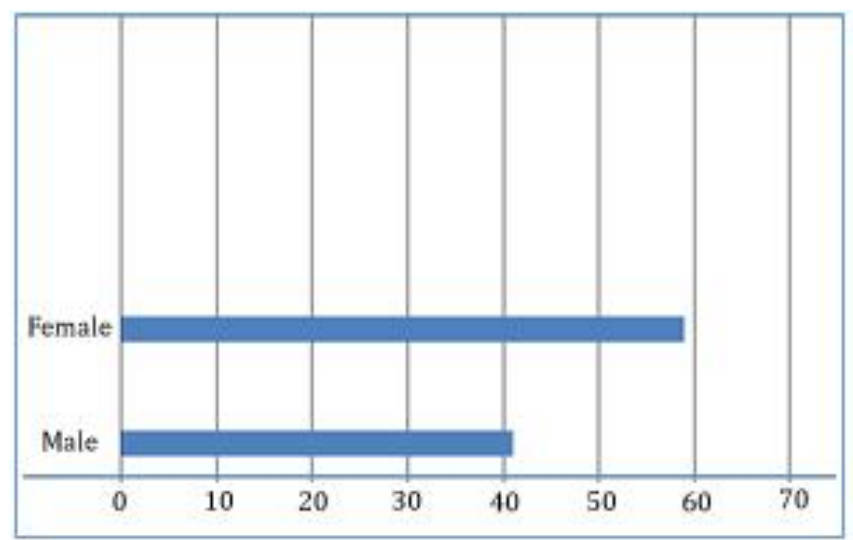

Graph 1. Distribution of Gender Female (59\%) - Male (41\%)

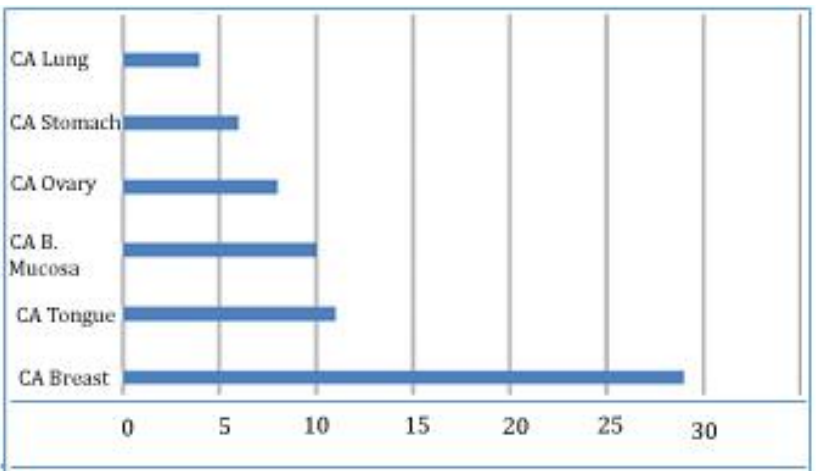

Graph 2. Type of Cancer

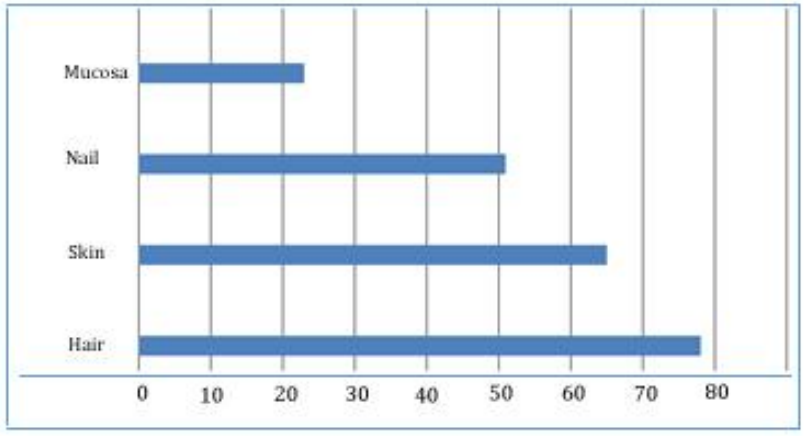

Graph 3. Frequency of adverse effect Hair (78\%) > Skin (65\%) > Nail (51\%) > Mucosa (23\%)

Hair changes were mainly in the form of anagen effluvium seen in 156 (78\%) patients [Figure 1].

The skin changes were supravenous hyperpigmentation [Figure 2], generalised pruritis, facial pigmentation, acneiform (Papulopustular) eruptions, xerosis, hyperpigmentation of palm and sole, and toxic epidermal necrolysis, acral erythema, hand-foot syndrome, extravasation, erythema nodosum and flagellate dermatitis.

The most common nail finding observed was melanonychia [Figure 3], which was seen in 74 (37\%) patients followed by onychoschizia [Figure 4], Muehrcke's lines, Mees' lines and Beau's lines.

Mucosal changes included pigmentation of tongue [Figure 5], oral ulcer, gingivitis [Figure 6] and stomatitis.

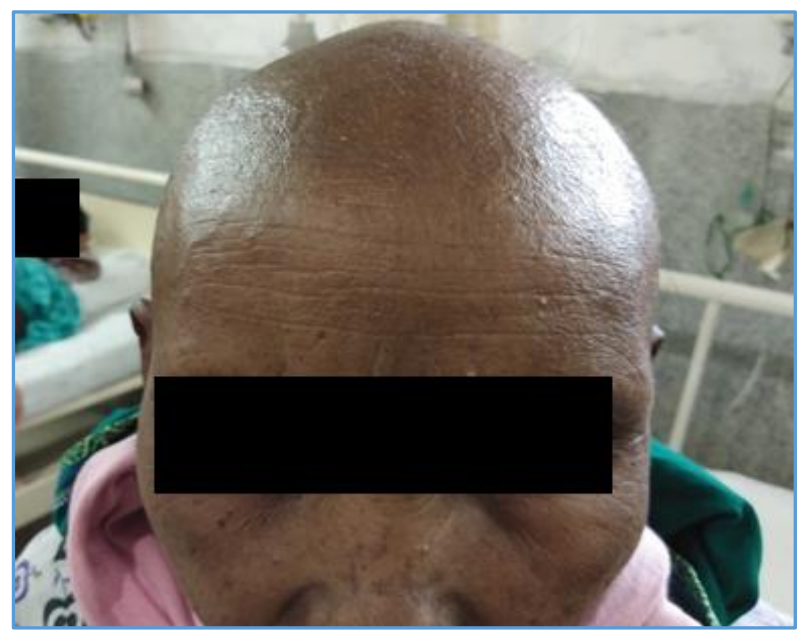

Figure 1. Anagen Effluvium 


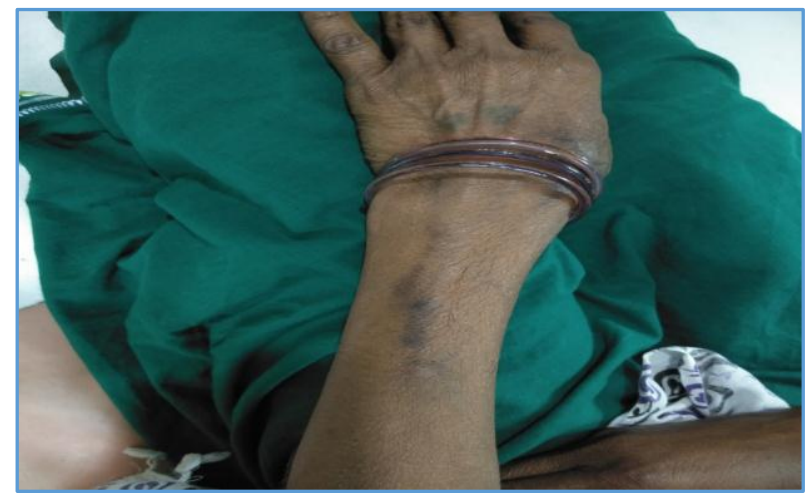

Figure 2. Supravenous Hyperpigmentation

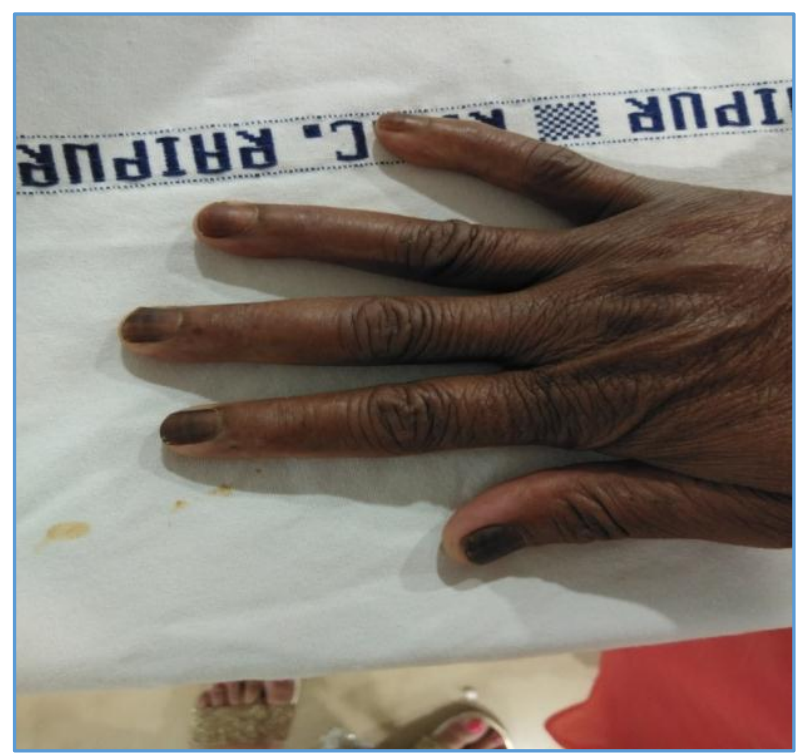

Figure 3. Diffuse Melanonychia

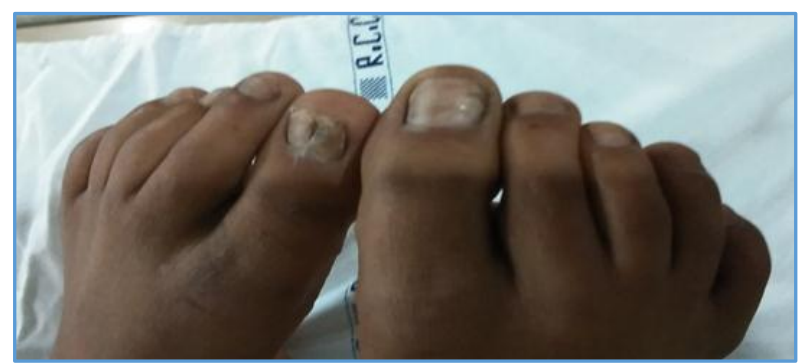

Figure 4. Onychoschizia

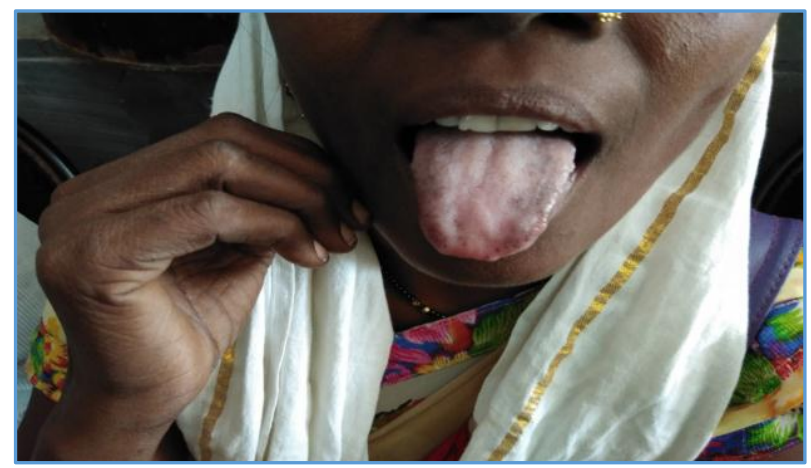

Figure 5. Black Pigmentation of Tongue

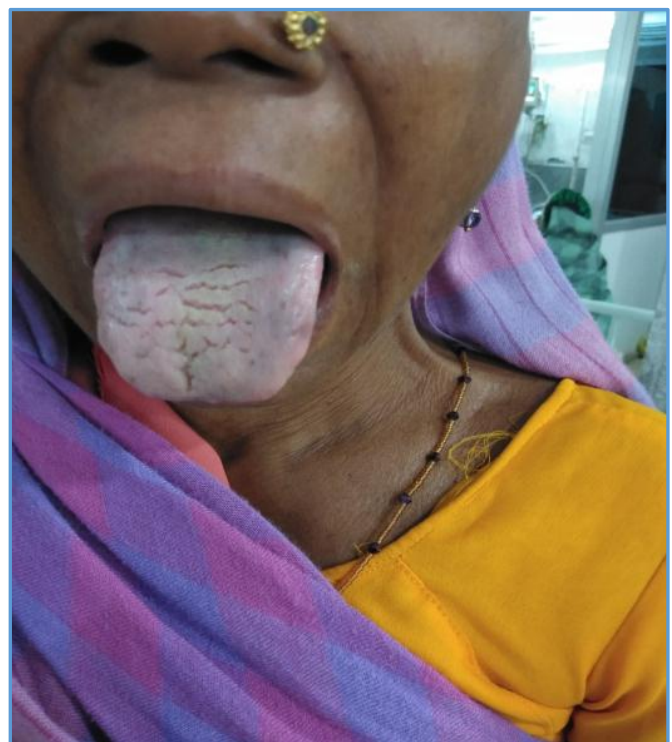

Figure 6. Glossitis and Furrowed Tongue

\section{DISCUSSION}

Newer chemotherapy agents having specific targets in the pathogenesis of malignancies and older agents are associated with a variety of cutaneous adverse events. These drugs have a small therapeutic index and thus may be toxic to the rapidly proliferating cells of the skin, hair and nails at therapeutic doses resulting in a wide array of cutaneous side effects.[1],[6]

In our study among all the cutaneous manifestation, most common ones were anagen effluvium followed by supravenous hyperpigmentation, xerosis and melanonychia of nail.

Hair loss has been rated as one of the most distressing side effects of chemotherapy along with vomiting and nausea.[7] There have been reports of refusal of chemotherapy, especially among women, because of the risk of hair loss. Alopecia was the first most common adverse effect in our study [156 (78\%) patients] similar to the study done by Kamil et al and Chiewchanvit et al.[8] The mechanism involved is a sharp fall in mitotic activity of the matrix cells of the hair follicle due to cytotoxicity. The cessation of mitotic activity in the hair matrix results in a narrow weakened portion of the hair shaft known as Pohl-Pinkus constriction, which is prone to fracture. [9]

Hair loss was seen within the first 1 month after the onset of chemotherapy in our study, which was also observed in a study by Chadha et al.[10] Anagen effluvium is the most common cause of hair loss associated with anti-cancer drugs and it usually begins in 1 - 2 weeks after starting the drug, becoming more apparent in the subsequent $4-8$ weeks. Drugs like doxorubicin, daunorubicin, docetaxel and cyclophosphamide are more likely to cause anagen effluvium. In our study, hair loss was seen in patients on paclitaxel and carboplatin, daunorubicin, cyclophosphamide and vincristine as noted in previous reports.

The severity of alopecia is directly related to the dosage, duration and regimen of therapy. Permanent hair loss is reported with certain drugs such as cyclophosphamide and busulfan.[11] Minoxidil was found to be ineffective in preventing chemotherapy-induced hair loss.[12] Hair loss is almost always reversible with good regrowth seen after cessation of treatment.[12] It is therefore important to 
reassure the patient regarding regrowth of hair after completion of treatment.

Hyperpigmentation has been reported to occur with anticancer drugs, which may be in the form of diffuse or localised involvement of skin, mucosa or nails. The mechanism remains unknown, but it is postulated to be due to accumulation of drug in skin or a direct toxic effect on melanocytes stimulating increased melanin production or elevated adrenocorticotropic hormone and melanocyte stimulating hormone. The drugs commonly causing pigmentation are cyclophosphamide, hydroxyurea, doxorubicin, cisplatin, fluorouracil, etoposide, busulfan and bleomycin.[1]

Along with cutaneous pigmentation, longitudinal and transverse bands or diffuse nail pigmentation and patchy pigmentation of tongue and buccal mucosa may also occur.

In our study we saw generalised hyperpigmentation of most of the body, both covered and exposed skin areas, results being consistent with some previous studies.[13,14]

Xerosis was seen in $26 \%$ patients. Majority of them (53.9\%) noticed skin lesions by $4-6^{\text {th }}$ week of therapy. Abnormal keratinocyte differentiation, which leads to an impaired sebaceous gland function and loss of ability to retain water may be the cause of xerosis.[15] $\mathrm{A}$ similar incidence of xerosis (22.2\%) was reported by Pavey et al, but the onset of lesions was noticed earlier at 2 - 4 weeks.[14]

Fabbrocini et al reported an incidence of $41.7 \%$ of xerosis in his study.[16] The drugs found to cause xerosis in this study were cyclophosphamide, carboplatin, ifosfamide, mesna and vincristine. Liberal use of emollients and general hydrating measures should be explained to the patients to minimise this side effect.[16]

Among nail changes, melanonychia was the most frequently seen adverse event and occurred in $15 \%$ cases followed by Beau's lines in 8\% patients and Mees' lines in $4 \%$ cases. Paronychia and loss of nail were the complaints in $2 \%$ and $1 \%$ patients, respectively.

\section{CONCLUSION}

Oncological therapies have become more selective and have low systemic toxicity because of their high specificity, but cutaneous side effects are common and may worsen the quality of life of these patients. Our observations necessitate a joint effort between dermatology and oncology for the early recognition and adequate treatment of the cutaneous adverse effects associated with cancer chemotherapy, which may help in reducing morbidity and improving compliance.

\section{REFERENCES}

[1] Susser WS, Whitaker-Worth DL, Grant-Kels JM. Mucocutaneous reactions to chemotherapy. J Am Acad Dermatol 1999;40(3):367-98.
[2] Joshi SS, Ortiz S, Witherspoon JN, et al. Effects of epidermal growth factor receptor inhibitor-induced dermatologic toxicities on quality of life. Cancer 2010;116(16):3916-23.

[3] Kamil N, Kamil S, Ahmed SP, et al. Toxic effects of multiple anticancer drugs on skin. Pak J Pharm Sci 2010;23(1):7-14.

[4] Jatoi A, Nguyen PL. Do patients die from rashes from epidermal growth factor receptor inhibitors? A systematic review to help counsel patients about holding therapy. Oncologist 2008;13(11):1201-4.

[5] Wagner LI, Lacouture ME. Dermatologic toxicities associated with EGFR inhibitors: the clinical psychologist's perspective. Impact on health-related quality of life and implications for clinical management of psychological sequelae. Oncology (Williston Park) 2007;21(11 Suppl 5):34-6.

[6] Hasnat A, Poddar S, Sultana R, et al. Pattern of adverse drug reactions due to cancer chemotherapy in tertiary care teaching hospital in Bangladesh. Dhaka Univ J Pharm Sci 2010;8:11-6.

[7] Yeager CE, Olsen EA. Treatment of chemotherapyinduced alopecia. Dermatol Ther 2011;24(4):432-42.

[8] Chiewchanvit S, Noppakun K, Kanchanarattanakorn K. Mucocutaneous complications of chemotherapy in 74 patients from Maharaj Nakorn Chiang Mai Hospital. J Med Assoc Thai 2004;87(5):508-14.

[9] Hinds G, Thomas VD. Malignancy and cancer treatment-related hair and nail changes. Dermatol Clin 2008;26(1):59-68.

[10] Chadha V, Shenoi SD. Hair loss in cancer chemotherapeutic patients. Indian $\mathrm{J}$ Dermatol Venereol Leprol 2003;69(2):131-2.

[11] Guillot B, Bessis D, Dereure O. Mucocutaneous side effects of antineoplastic chemotherapy. Expert Opin Drug Saf 2004;3(6):579-87.

[12] Hammond-Thelin LA. Cutaneous reactions related to systemic immune modulators and targeted therapeutics. Dermatol Clin 2008;26:121-59, ix.

[13] Pavey RA, Kambil SM, Bhat RM. Dermatological adverse reactions to cancer chemotherapy. Indian J Dermatol Venereol Leprol 2015;81(4):434.

[14] Garg T, Sanke S, Yadav P, et al. Mucocutaneous manifestations in patients on chemotherapy with pediatric haematological malignancies. Astrocyte 2016;3(2):74-7.

[15] Fabbrocini G, Cameli N, Romano MC, et al. Chemotherapy and skin reactions. J Exp Clin Cancer Res 2012;31:50.

[16] Lacouture ME, Boerner SA, Lorusso PM. Non-rash skin toxicities associated with novel targeted therapies. Clin Lung Cancer 2006;8 Suppl 1:S36-42. 